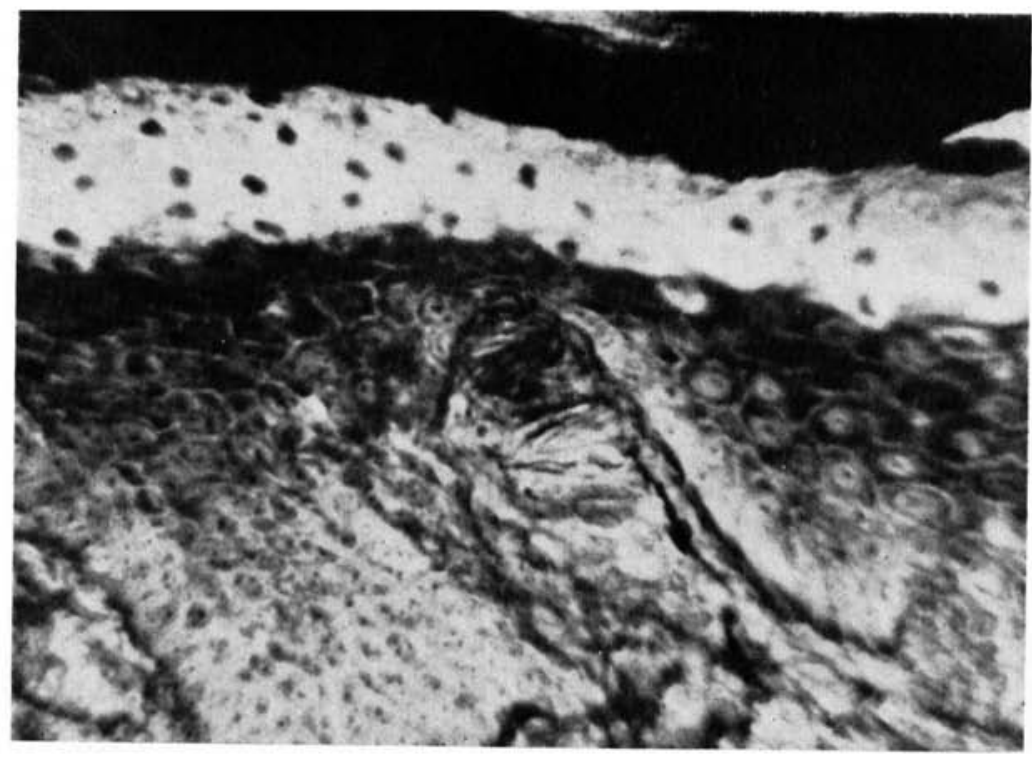

Fig. 1. Meissner's Corpuscle in dermal papillae showing a single nerve fibre ascending into the corpuscle and ramifying with fragmented fine filaments. Distal pad of ring finger, Male, 32 years, Lepromatous $x 400$

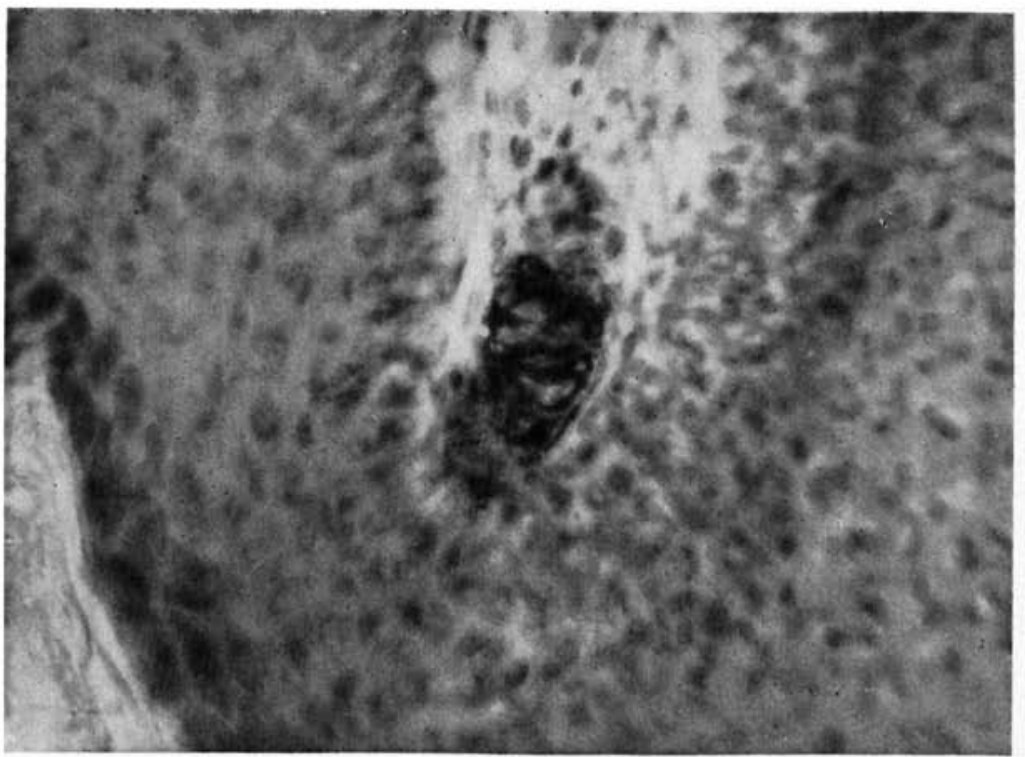

Fig. 2: Corresponding section to Fig.l showing positve Cholinesterase reaction in Meissner's Corpuscle Cholinesterase $x 400$ 


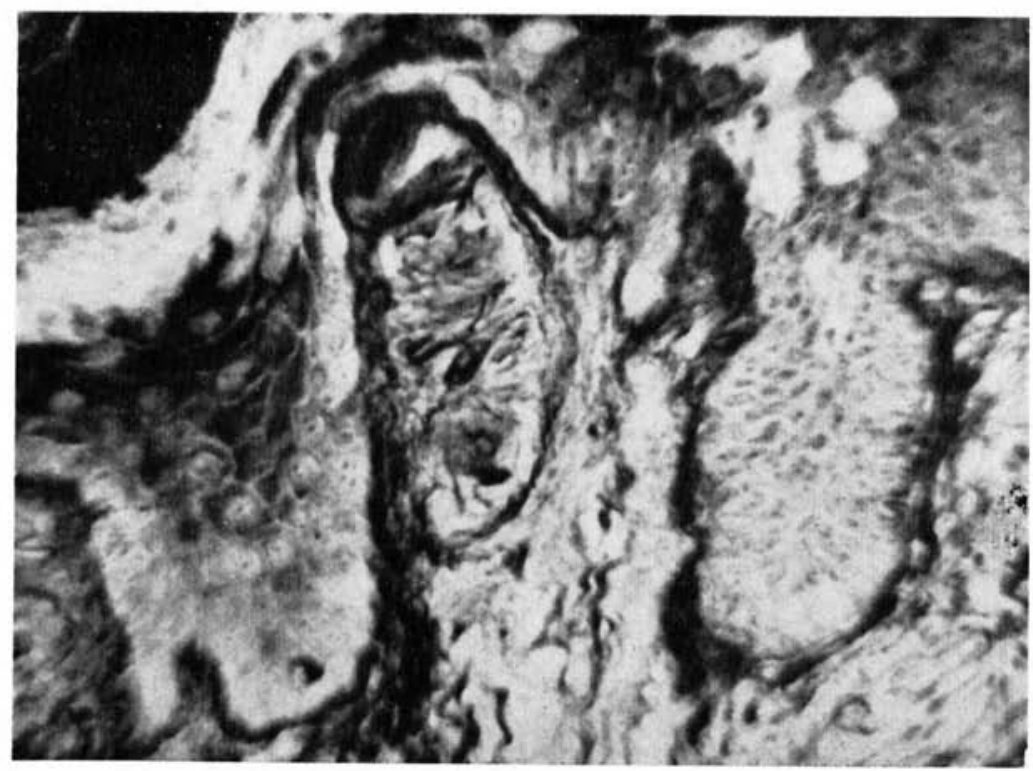

Fig. 3. Meissner's Corpuscle showing fine filament ramifying the Corpuscle and a nerve fibre is seen ending in papillary ridge. Distal pad of middle finger, male 25 years. Early leptomatous leprosy $x 400$

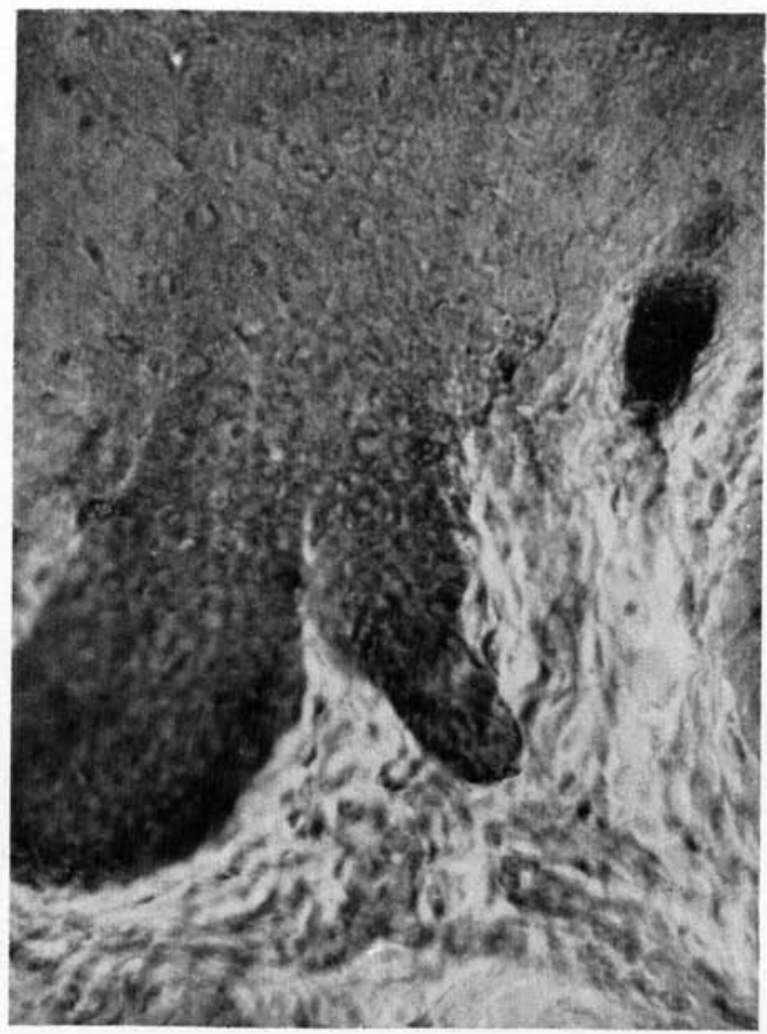

Fig. 4. Corresponding section to Fig. 3, showin ositive cho- 


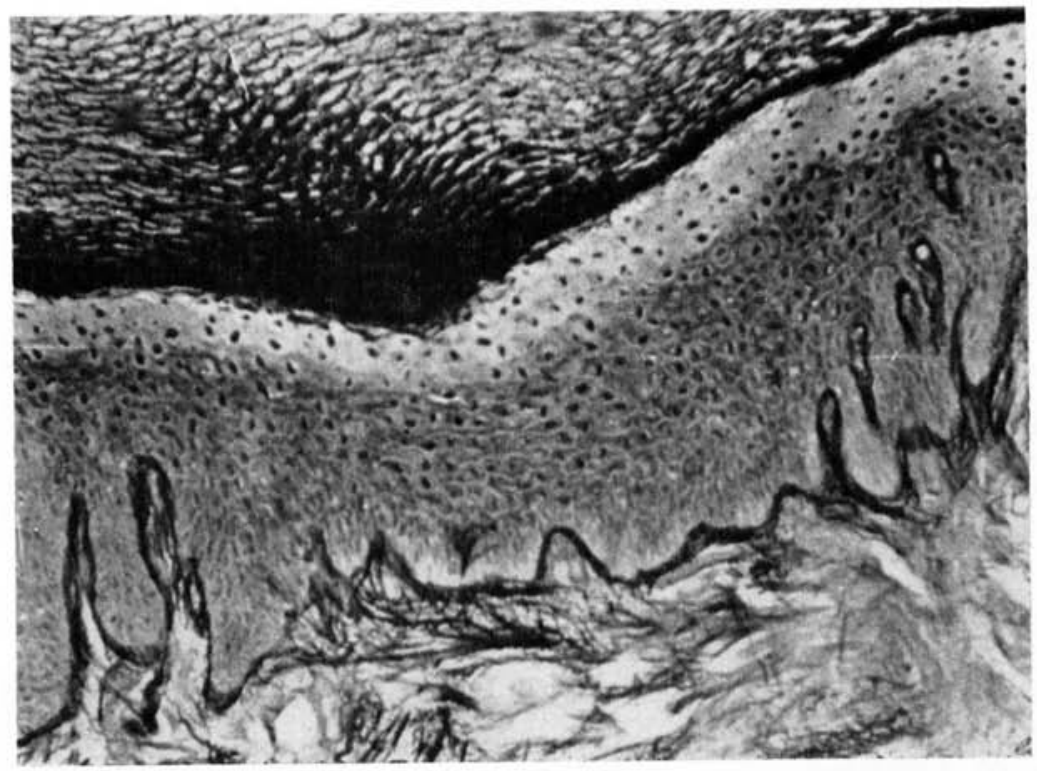

Fig. 5. Section of digital skin showing the epithelium with flattened papillary ridges. Distal pad of middle fiinger, male, 27 vears. Tuberculoid $x$ 100)

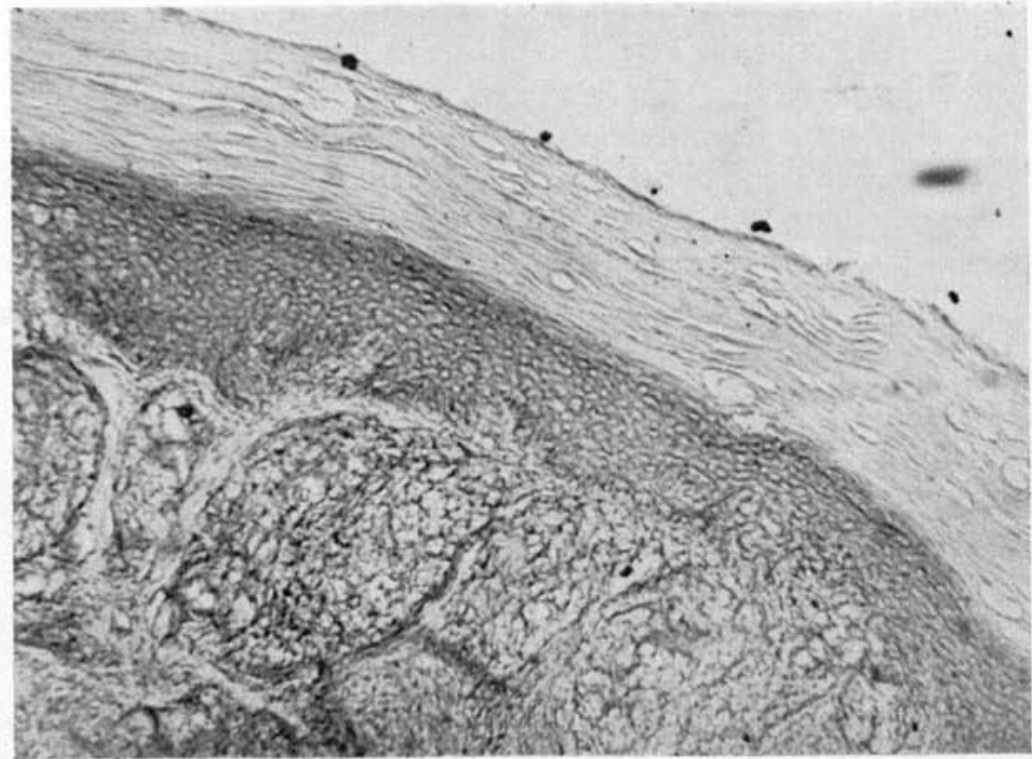

Fig. 6. Corresponding section to Fig. 5, of another tuberculoid patient showing no cholinesterase activity' in any part of the epithelium, Cholinesterase $x 1(0)$ 


\section{STUDIES ON THE DISTRIBUTION OF CHOLINESTERASES IN RELATION TO NEURO-HISTOLOGICAL CHANGES IN SPECIALIZED SENSORY NERVE ENDINGS IN LEPROSY SKIN}

by A. Paul Jayaraj, F.R.M.s.

(Central food Techmological Research Institute, Mysore, India)

It is generally accepted that the nervous transmission is a chemical process and depends upon the action of cholinesterases during stimulation. The cholinesterase system which involves the enzymatic hydrolysis of acetyl choline plans an important role in the mechanism of transmitting impulses at nerve junction and nerve endings. The precise significance of this enzyme in nervous activity is not fully understood. NACHMANSON (1959) suggests that this enzyme is essential not only in synoptic activities but also in nervous conduction. In general it is believed that cholinesterase has an active function in nervous transmission to restore the mechanism for repeated activity. CaUna (1960) worked out on the cytological localisation of the enzyme in relation to the structure of the cutaneous receptors compared to neurohistological studies and he suggests the enzyme cholinesterases may constitute an active agent in synoptic transmission of nervous impulse in Meissner's corpuscles.

The present work is an investigation on the distribution of cholinesterase compared to neuro-histological changes in Meissner's corpuscles and some of the specialised sensory nerve endings in leprosy skin. The presence of high concentration of cholinesterases in specialised sensory nerve endings prompted us to examine the distribution of cholinesterase in the skin of leprosy patients. Meissner's corpuscles are concerned with selective touch receptors and are designed for tactile discrimination.

\section{Material and Methods}

Twenty piece of skin were taken from the distal pad of the fingers from 20 patient howing typical lesions of leprosy; 15 from lepromatous and 5 from tuberculoid cases. There were no visible lesions anywhere on the finger pads. Biopsies were also taken from lesions of each patient to confirm histologically the type of lesion. Tissues were fixed in $10 \%$ neutral formalin. Half of the specimen was immediately transferred to ice for histochemical studies. Out of the remaining tissue. frozen sections were taken at 20 micron thickness and stained for nerve fibres by the method described by BALASUbramanyan. JayaraJ and Gass (1954). Twenty sections were taken from each specimen for this study. Remaining tissues were processed for paraffin sections. Sections were stained for hematoxylin and eosin 
and for acid fast bacilli by the method described by JAYARAJ (1955). Three pieces of normal skin were taken from digital pad of the fingers as control.

Pieces of skin intended for histochemical studies were fixed in $10 \%$ cold formalin at 4 degree centigrade for four hours. Frozen section at 20 microns were taken and incubated for 30 minutes to 1 hour using histochemical technique of KoELLE (1951).

\section{Results}

In lepromatous leprosy the area occupied by Meissner's corpuscle shows mild to moderate intensity of cholinesterase activity. Superficial endings also show scattered activity of the enzyme. Mild intensity of the enzymatic reaction is seen in the papillary nerve endings. The nerve bundles did not show cholinesterase activity. The enzymatic activity differed in intensity from one to another in Meissner's corpuscles. In early lepromatous leprosy the intensity of enzymatic activity seems to be more concentrated in Neissner's corpuscles. The reaction on the papillary nerve endings also shows heavier concentration. In tuberculoid leprosy, the cholinesterase activity is not seen. The enzymatic reaction on the epidermal region shows no activity. The papillae that occupy the Meissner's corpuscle are completely collapsed and compressed. Practically there are no papillary ridges seen and the enzymatic activity is absent.

The neuro-histological changes in Meissner's corpuscles in lepromatous leprosy show a certain complexity and fragmentation of axons. The corpuscles receive one to two myelinated nerve fibres which branch as fine filaments in corpuscles. An occasional single myelinated nerve fibre is seen ramifying in the Meissner's corpuscles. The whole process looks myelinated. In few cases Meissner's corpuscles show the free fading filaments. In early lepromatous leprosy the Meissner's corpuscles look almost normal. The ramification of the nerve filaments is well seen. Paraffin sections stained for acid fast bacilli show abundant bacilli alongside the neuro-fibrillary ramification. In lepromatous leprosy, the epithelium is flattened leaving the surfaces of the papillary ridges in limited length in the corium. The ridges are prominently seen even though the epithelium is flattened. The nerve fibres ascending from the corium are seen ending in the papillary ridges. The ridges are found to vary in length between advanced lepromatous to early lepromatous leprosy. The paraffin sections stained for acid fast bacilli show higher concentration of bacilli in the papillary ridges. In tuberculoid leprosy, the thickness of the epithelium is reduced. In many areas, there are no papillary ridges seen. The dermal papillae that occupies the Meissner's corpuscle is compressed. In no part of the epithelium the nerve endings are seen. 


\section{Discussion}

It is well known that there is high concentration of cholinesterases in the specialized sensory nerve endings, even though the function of this particular enzyme in these locations is still unknown. FLOREY (1961) introduced a theoretical consideration concerning the widely accepted chemical transmission of the nerve impulse in the nerve endings. He states that axons and nerve endings contain the enzyme system capable of synthesizing the chemical transmitter which is stored in physiologically inactive bound form and the transmitter is released by the nerve endings upon the arrival of the nerve impulse. Cauna (1960) suggests that in Meissner's corpuscles the mechanical stimulus is converted into a nerve impulse within the cytoplasm of the laminar cell, and microvesicles, produced by the perinuclear cytoplasm, are carriers of an excitatory agent, probably acetyl choline. It is of considerable interest to observe that in leprosy the enzymatic reaction of cholinesterases is distributed wherever nerve endings are distinctly seen. In lepromatous leprosy the intensity of the reaction differed from one Meissner's corpuscle to another. It is felt that the intensity of the reaction depends upon the severity of damages caused to the fine nerve filaments by the disease process. When the dermal papillae is compressed and the corpuscle is destroyed, the enzymatic reaction is completely absent. This clearly shows that the nerve endings are not capable of synthesizing the enzyme system when they are damaged. GOMORI (1956) stated most nerve fibres will not be demonstrated by any enzymatic technique now available and even in case of cholinergic nerves, it appears that only a part of them, usually the endings, are positive. In leprosy it was found that only the endings in Meissner's corpuscles and Merkel tactile discs show the enzymatic activity. The intensity of the reaction also depends upon the damage caused to the nerve endings. The nerve bundles deep in the corium have not shown enzymatic reaction. JAYARAJ and Chaudhury $(1961,1962)$ studied the neuro-histological changes in Meissner's corpuscles in leprosy and the structure and function of the papillary ridges in leprosy skin. They found that the terminal fibres in Meissner's corpuscles undergo characteristic changes in advanced lepromatous leprosy and in early lepromatous leprosy the neuro-fibrillary ramification looks almost normal. They also found extensive nerve fibres embedded in the papillary ridges of the lepromatous skin and in tuberculoid leprosy the ascending nerve fibres are mostly destroyed resulting in severe sensory impairment. Compared to the above neuro-histological changes, in the present investigation of the distribution of cholinesterases in nerve endings, it is found that the intensity of the reaction is reduced wherever the nerve terminals are damaged or the dermal papillae are compressed destroying the Meissner's corpuscles. The flattened epithelium without ridges, which is commonly found in the tuberculoid type of 
leprosy and where the nerve endings are not found, the enzymatic reaction of cholinesterases is completely absent. Whether the enzyme has any role to play in the conduction of nerve impulse or not, it is of considerable interest to note that the enzyme cholinesterases are present wherever the fine nerve is ending in the epithelium of leprosy skin.

\section{Summary}

1. Biopsies from the distal pad of the fingers from 20 leprosy patients comprising 15 lepromatous and 5 tuberculoid were studied by cytological, nerve staining and histochemical methods.

2. It was found that the cholinesterase reaction is reduced in Meissner's corpuscles and in papillary ridges in advanced lepromatous leprosy where the terminal nerve fibres undergo characteristic changes. In early lepromatous leprosy the Meissner's corpuscles looked almost normal and intensity of the cholinesterase reaction was comparatively increased.

3. In tuberculoid leprosy the dermal papillae that occupy the Meissner's corpuscle seemed to be compressed resulting in the destruction of Meissner's corpuscles and the papillary ridges are found to be flattened. The cholinesterase activity is completely absent in all parts of the epithelium.

4. Even though the final agreement is lacking in details on the role of cholinesterases in nervous activity, there is evidence to indicate that it may serve to maintain the structure of the specialised sensory nerve endings for repeated nerve impulse in leprosy skin.

\section{Acknowledgement}

The author is highly grateful to Dr. V. Subrahmanyan, D.SC., F.R.I.C., F.N.I., Director, Central Food Technological Research Institute, Mysore, for kindly offering facilities and encouragement to this investigation and to Dr. T. S. Sambasivan, Associate Professor of Dermatology, Venerology and Leprosy, Mysore Medical College, for kindly providing the clinical material for this investigation. The author is also indebted to Dr. M. Swaminathan, D.SC., F.N.I., Assistant Director, Central Food Technological Research Institute for kind and valued encouragement. The author is highly grateful to the late Dr. George Gomori for kindly sending the gift sample of substrate which was used for histochemical investigation.

\section{References}

NaChmansons, D. (1959) Chemical and Molecular basis of nerve activity, London, (Academic Press).

CAUnA, N. (1960) The Distribution of Cholinesterases in the cutaneous receptor organs, especially touch corpuscles of the human fingers. J. Histochemistry Cytochemistry, 8, 367-375. 
Balasubramanynan, M., Jayaraj, A. P. and Gass, .H. H. (1954) An Improved Histological Method for the Examination of Cutaneous Nerves in Leprosy. Leprosy Rev., 15, 83-86.

JayaraJ, A. P. (1955) Periodic acid in the staining of Acid fast bacilli in Tissue Section, J. Anat. Soc. India, 4, 41-42.

Koelle, G. B. (1951) The elementation of enzymatic diffusion artifacts in the Histochemical localization of cholinesterases and a survey of their cellular distribution. J. Pharmacol, 103, 153-171.

Florey, E. (1961) Comparative Physiology: Transmitter Substances, Annual Review of Physiology, 23, 501-528.

GOMORI, G. (1956) personal communication.

JayaraJ, A. P. and Chaudhury, D. S. (1961) Studies on the Neuro-Histological changes in Meissner's Corpuscles in leprosy. Leprosy Rev., 32, $153-157$.

JayaraJ, A. P. and Chaudhury, D. S. (1962) Studies on the structure and function of the papillary ridges of digital skin in leprosy. Leprosy Rev., 33, $41-44$. 